\title{
GÊNERO Calophyllum: IMPORTÂNCIA QUÍMICA E FARMACOLÓGICA
}

Vânia Floriani Noldin, Daniela Buffon Isaias e Valdir Cechinel Filho*

Núcleo de Investigações Químico-Farmacêuticas, Centro de Ciências da Saúde, Universidade do Vale do Itajaí, CP 360, 88302-202 Itajaí - SC, Brasil

Recebido em 4/1/05; aceito em 3/8/05; publicado em 8/2/06

\begin{abstract}
Calophyllum GENUS: CHEMICAL AND PHARMACOLOGICAL IMPORTANCE. The Calophyllum genus (Clusiaceae) is composed of about 200 species, with a pantropical distribution. Some species are medicinal and are used against several diseases, including gastric ulcers, infectious pathologies, painful, inflammatory processes and as molluscicidal. A search in the literature regarding the chemical and biological aspects of these plants indicates cytotoxic activity against several cell lines, inhibition of HIV-1 reverse transcriptase, antisecretory and cytoprotective properties, antinociceptive, molluscicidal and antimicrobial effects, among others, related particularly to the presence of coumarins, xanthones, flavonoids, and triterpenes.
\end{abstract}

Keywords: Calophyllum; phytochemical study; pharmacological study.

\section{INTRODUÇÃO}

As plantas são utilizadas desde os primórdios da civilização para tratamento e cura de enfermidades, o que propiciou uma das bases mais importantes para o nascimento da medicina. Estas têm contribuído significativamente para o fortalecimento da indústria farmacêutica, através do isolamento de substâncias bioativas, cuja complexidade de muitas estruturas químicas inviabilizaria técnica e economicamente a síntese orgânica de tais substâncias ${ }^{1-4}$.

Muitas das propriedades terapêuticas das plantas são relatadas pela população, as quais são confirmadas em sua maioria nos estudos científicos, comprovando a importância da pesquisa etnofarmacológica. Tais propriedades propiciaram o desenvolvimento de vários medicamentos, sejam estes obtidos por síntese a partir de molécula protótipo ou através de isolamento, algumas vezes, biomonitorado ${ }^{3-7}$.

Devido a todos estes aspectos, vê-se um interesse crescente na utilização e pesquisa de plantas medicinais, objetivando fins terapêuticos, aliadas à boa aceitabilidade destes produtos no mercado farmacêutico e as altas cifras que circundam a comercialização de fitomedicamentos, observada na última década ${ }^{3,4,8-11}$.

O Brasil é o país com maior potencial para pesquisa com espécies vegetais, pois detém a maior e mais rica biodiversidade do planeta, distribuída em seis biomas distintos. Um dos gêneros que vem despertando grande interesse na comunidade científica, devido aos promissores resultados químicos e biológicos, é o Calophyllum (Clusiaceae). Este gênero é composto por grande grupo de árvores tropicais, com aproximadamente 180-200 espécies restritas aos trópicos quentes e úmidos, sendo algumas espécies de ocorrência predominante no Brasil, como por ex., a Calophyllum brasiliense Camb., distribuída principalmente na Mata Atlântica e no Cerrado. Muitas destas espécies são empregadas na medicina popular para tratar injúrias como dor, processos infecciosos, inflamatórios e úlceras ${ }^{12,13}$.

As principais partes utilizadas são as folhas ou cascas do caule, sob forma de chás. Quanto à quimiossistemática, as plantas pertencentes a este gênero consistem principalmente de cumarinas, xantonas, esteróides, triterpenos e biflavonóides, sendo que muitas destas substâncias são dotadas de ações biológicas pronunciadas e importantes ${ }^{14-18}$.

Este trabalho descreve as principais espécies de Calophyllum já estudadas e descritas em literatura, com foco especial em seus

*e-mail: cechinel@univali.br principais constituintes químicos e propriedades biológicas. Devido à grande quantidade de moléculas isoladas relatadas para o gênero Calophyllum, são apresentadas algumas estruturas de substâncias inéditas e/ou com potencial terapêutico evidenciado.

\section{ASPECTOS QUÍMICOS E BIOLÓGICOS DAS ESPÉCIES DE Calophyllum}

\section{Calophyllum brasiliense}

As árvores de $C$. brasiliense podem chegar a $40 \mathrm{~m}$ de altura e 1 a $3 \mathrm{~m}$ de diâmetro, sendo sua madeira utilizada para construções, pisos e mobílias. Esta planta é comumente conhecida no Brasil como guanandi ou guarandi. Suas cascas são utilizadas para tratamento de reumatismos, varicoses, hemorróidas e úlceras, e as folhas, para processos inflamatórios ${ }^{14,18,19}$.

Quanto às atividades biológicas relatadas na literatura, esta é uma das espécies do gênero mais estudada, destacando-se as propriedades antibacterianas, antifúngicas, citotóxicas, inibitória da promoção de tumor, inibitória da replicação do HIV-1 IIIb/LAV dos extratos e frações das folhas, caules e raízes ${ }^{14,20-25}$.

Da resina do tronco foi isolada uma goma ácida esverdeada, consistindo na sua totalidade de dois compostos isoméricos, ácido brasiliênsico (1) e ácido isobrasiliênsico (2) ${ }^{26}$.

Silva e colaboradores ${ }^{18}$ analisaram a composição química das folhas, sendo que da fração acetato de etila obtiveram cinco sólidos puros, identificados como quercetina, ácido gálico, ácido protocatético, hiperosídeo e amentoflavona.

Avaliando-se os constituintes majoritários das diferentes partes da planta, detectou-se que a composição química é distinta para cada parte da planta. Das raízes foram isoladas as substâncias 1,5diidroxixantona, friedelina e ácido betulínico, enquanto nos frutos os principais compostos são ácido gálico e ácido protocatético, e em menor proporção o hiperosídeo. Nas flores há predominância de ácido protocatético e $( \pm)$ epi-catequina ${ }^{27}$.

Os extratos e frações de folhas, flores, frutos e raízes desta espécie, avaliados no teste de contorções abdominais induzidas por ácido acético em camundongos, demonstraram significativo efeito antinociceptivo, assim como os compostos isolados da fração acetato de etila das folhas (quercetina e amentoflavona) e da fração clorofórmica das raízes (friedelina e 1,5 diidroxixantona). No mo- 
delo da formalina, apenas as frações das folhas e raízes exibiram atividade antinociceptiva em relação à segunda fase (dor de origem inflamatória), mas foram praticamente inativos em relação à primeira fase (dor de origem neurogênica) ${ }^{18,27}$.

O extrato bruto e frações polares (não solúveis em clorofórmio) e não polares (solúveis em clorofórmio) de diferentes partes da planta (raízes, caules, folhas, flores e frutos) apresentaram atividade contra bactérias Gram-positivas e sobre algumas cepas Gram-negativas, sendo que o ácido protocatético foi efetivo contra todos os microrganismos testados e a 1,5-diidroxixantona somente contra Gram-positivas ${ }^{25}$.

Estudos preliminares com as cascas do caule da C. brasiliense demonstraram atividade gastroprotetora em modelos de úlcera induzidos por etanol, indometacina e estresse por frio ${ }^{19}$.

Em um estudo biomonitorado com os extratos metanólico, hexânico e acetônico, obtidos dos galhos desta planta, foram isoladas as dipiranocumarinas calanolideo A, B, C (3-5) e soulattrolideo (6), e outras substâncias como friedelina, ácido isoapetálico, canofilol e amentoflavona. As substâncias 3, 4 e $\mathbf{6}$ apresentaram efeitos promissores inibitórios sobre a enzima transcriptase reversa do vírus HIV-124.

Das folhas foram isolados derivados cumarínicos do tipo mammea (mammea $\mathrm{A} / \mathrm{BA}, \mathrm{A} / \mathrm{BB}, \mathrm{B} / \mathrm{BA}, \mathrm{B} / \mathrm{BB}$, isomammeigina (711), mammea $\mathrm{C} / \mathrm{AO}, \mathrm{C} / \mathrm{OB}, \mathrm{B} / \mathrm{BA}$ ciclo $\mathrm{F}, \mathrm{B} / \mathrm{BB}$ ciclo $\mathrm{F}$ e outros). Esta classe de substâncias é caracterizada pela presença de esqueleto cumarínico 5,7-dioxigenado, possuindo um radical fenila ou alquila ligado ao C-4, e substituintes acila ou prenila ligados ao C-6 ou C-8. Ocorre particularmente em plantas dos gêneros Mammea e Mesua (Clusiaceae), sendo rara em outros gêneros ${ }^{20}$. Apenas os derivados do tipo mammea A apresentaram efeitos antibacterianos, inibindo o crescimento de S. aureus, S. epidermidis e Bacillus subtilis. Neste mesmo estudo, foram relatadas atividades citotóxicas significativas destes derivados cumarínicos (7-11) contra três linhagens de células tumorais humanas (K562, U251 e PC3), além da inibição in vitro da enzima transcriptase reversa do vírus $\mathrm{HIV}-1^{20}$.

Das cascas da $C$. brasiliense, foram isoladas sete novas xantonas, identificadas como brasixantona A, B, C, D, E, F e G (1218), sob forma de um óleo amarelo, exceto a brasixantona $B$, que cristalizou como agulhas. Também foram isoladas dez xantonas conhecidas: toxiloxantona A; 6-desoxijacareubina; 3,8-diidroxi-1,2dimetoxixantona; 8-desoxigartanina; cudraxantona F; 4-hidroxixantona; 1,2-dimetoxixantona; piranojacareubina; garcinina $\mathrm{B}$ e latisxantona C. As substâncias brasixantona B, C e D apresentaram efeito inibitório sobre o vírus Epstein-Barr ${ }^{21}$.

Em outro estudo realizado pelos mesmos autores com as cascas do caule foram isoladas 3 novas cumarinas, denominadas de brasimarinas A, B e C que foram efetivas na inibição do vírus Epstein-Barr ${ }^{22}$. Recentemente, também foram isolados das cascas do caule 6 novos ácidos cromanonas (19-24), que demonstraram potencial antibacteriano, especialmente contra Bacilus cereus e Staphylococcus epidermitis ${ }^{23}$.

$\mathrm{O}$ relato mais recente de agentes anticancerígenos desta espécie é da substância denominada GUT-70 (5-metoxi-2,2dimetil-6-(2-metil-1-oxo-2-butenil)-10-propil-2H,8H-benzol [1,2-b;3,4-b'] dipiran-8-ona (25), uma cumarina tricíclica isolada da casca do caule. Essa substância inibiu 6 tipos de linhagens de células leucêmicas com concentração e tempo-dependentes, cuja $\mathrm{CI}_{50}$ variou entre 2-5 mM, induzindo a apoptose das células leucêmicas ${ }^{28}$.

Estudos realizados com folhas e galhos desta planta evidenciaram importante propriedade moluscicida, a exemplo de outras espécies deste gênero ${ }^{29}$. A principal substância bioativa, isolada através de estudo fitoquímico bio-direcionado, foi identificada como sendo a cumarina (-) mammea A/BB, apresentando após 24 h uma
$\mathrm{DL}_{50}$ de 0,67 ppm e $\mathrm{DL}_{90}$ de 1,47 ppm sobre o caramujo Biomphalaria glabrata ${ }^{30}$.
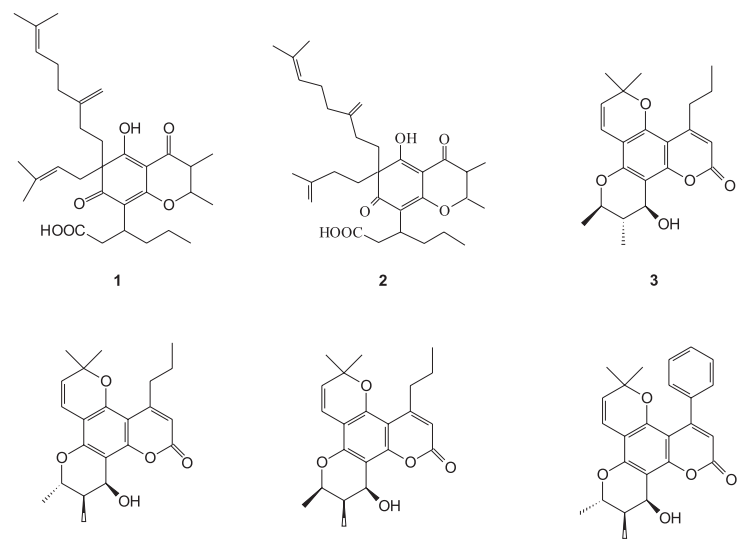

4<smiles>CC(C)=CCc1c(O)c(C(=O)CC(C)C)c2c(-c3ccccc3)cc(=O)oc2c1O</smiles>

5

6
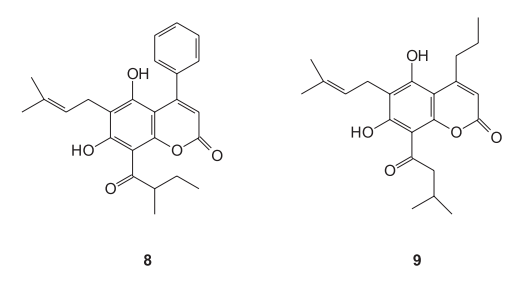

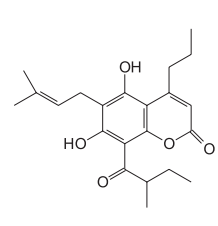

10

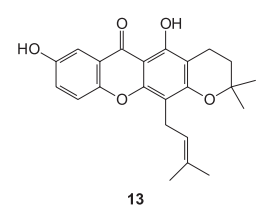

13

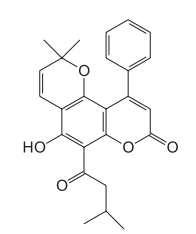

11
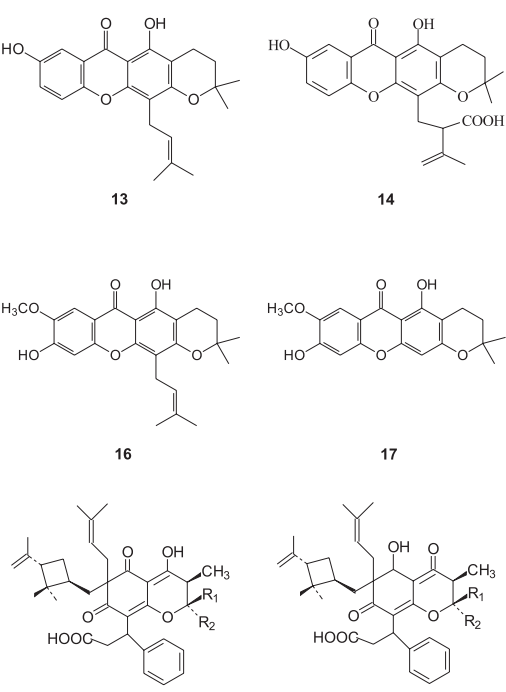

19, $\mathrm{R}_{1}=\mathrm{H}_{1} \mathrm{R}_{2}=\mathrm{CH}_{3}$ 20, $\mathrm{R}_{1}=\mathrm{CH}_{3}, \mathrm{R}_{2}=\mathrm{H}$

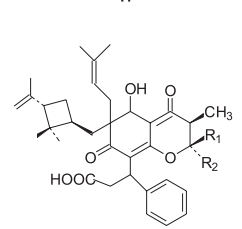

21, $\mathrm{R},=\mathrm{H}, \mathrm{R}_{2}=\mathrm{CH}_{3}$
22, $\mathrm{R}=\mathrm{CH}_{3}, \mathrm{R}_{2}=\mathrm{H}$

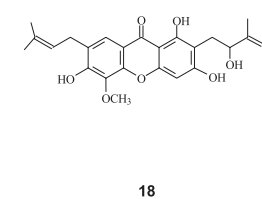

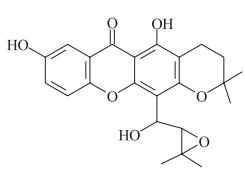

15

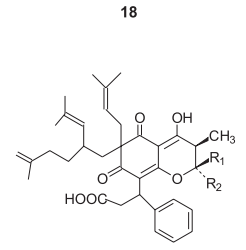

23, $\mathrm{R}_{1}=\mathrm{H}_{1} \mathrm{R}_{2}=\mathrm{CH}_{3}$
24, $\mathrm{R}_{1}=\mathrm{CH}_{3}, \mathrm{R}_{2}=\mathrm{H}$

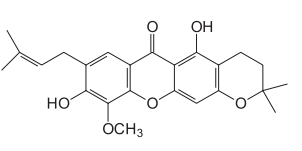

12

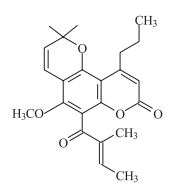




\section{Calophyllum papuanum}

Da casca do caule, dois compostos estereoisoméricos foram extraídos com pentano, e identificados como ácidos papuânico (26) e isopapuânico (27) ${ }^{26}$.

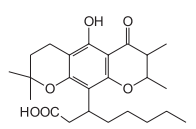

26

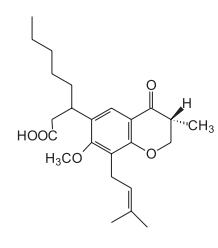

27

\section{Calophyllum fragrans}

A partir do extrato clorofórmico do tronco desta planta foram isoladas as xantonas: 6-desoxijacareubina (28), 1,5-diidroxi-6metoxixantona (29) e 1,7-diidroxixantona $(\mathbf{3 0})^{31}$.

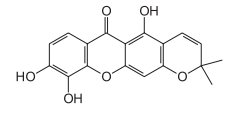

28

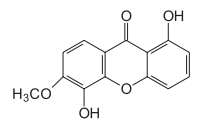

29

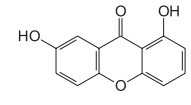

30

\section{Calophyllum inophyllum}

A $C$. inophyllum é utilizada com fins medicinais e ornamentais, sendo que são atribuídas a todas as suas partes propriedades terapêuticas, tais como o óleo das castanhas para reumatismos, gonorréia e sarna; a goma extraída do tronco para tratamento de feridas e úlceras; o decocto das cascas para tratar hemorragias internas e úlceras; além das indicações populares de atividades antisépticas, adstringente, expectorante, diurética e purgativa ${ }^{15,32}$.

Das raízes foram isoladas duas novas xantonas [caloxantonas A e B (31-32)], além das xantonas e flavonóides já conhecidos: macluraxantona, 1,5-diidroxixantona-6-desoxijacareubina; 2-3metilbut-2-enil-1,3,5-triidroxixantona; 2-3-metilbut-2-enil-1,3,5,6tetraidroxixantona; 1,3,5-triidroxi-6,6'dimetilpirano (2',3',6,7)-4(1,1-dimetilprop-2-enil)-xantona; macluraxantona; (-)epicatequina, amentoflavona, inofinona e isoinofinona ${ }^{15,33,34}$. Das folhas foram isolados canofilol e ácido canofílico ${ }^{15}$.

Itoigawa e colaboradores ${ }^{35}$ testaram algumas substâncias isoladas desta espécie em modelos antitumorais in vitro, destacando-se os derivados 4-fenil-cumarinas, aos quais são atribuídas atividades inibitórias para a enzima transcriptase reversa do HIV1. As substâncias testadas foram: 4 fenil-cumarinas tetracíclicas (Inofilum A, C, D, E) três tricílicas (calocumarina A, calofiloide, apetatolide), duas dimetilciclopropil (calocumarina B e C) e um ácido cinâmico fenólico (ácido isocalofílico), ressaltando-se que a calocumarina A (33) foi a substância que apresentou os resultados mais promissores.

Em estudo recente realizado com as raízes desta planta, foram isoladas várias xantonas e alguns derivados denominados inoxantona, caloxantona A e B, macluraxantona, 1,5-diidroxixantona, ácido calofínico, ácido brasiliênsico, ácido inofiloídico, friedelan-3-ona, calaustralina, calofilolida, inofilum C e E. Todas as substâncias apresentaram citotoxicidade contra células da linhagem KB e potencial atividade antibacteriana ${ }^{36}$. Ainda, das folhas, foram isolados 3 novos triterpenóides do tipo friedelano, denominados de ácido 3,4secofreidelan-3,28-dioico, ácido 27-hidroxiacetato canofílico e ácido 3-oxo-27-hidroxiacetato-friedelan-28-oico ${ }^{37}$.

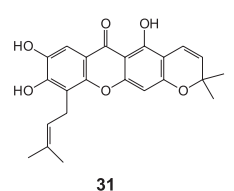

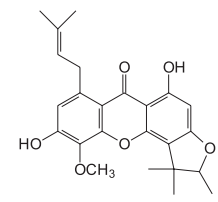

32

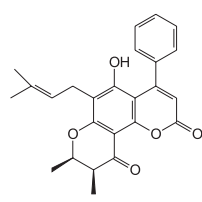

33

\section{Calophyllum dispar}

Da $C$. dispar foram estudadas as cascas do caule e os frutos, de onde foram isolados novos derivados de 4-fenilcumarinas como disparfuram B (34), disparacetilfuram A (35), mammea A/AA desidrociclo $F(36)$, mammea A/AA metoxiciclo $F(47)$, mammea A/ $\mathrm{BA}$ ciclo $\mathrm{F}$, mammea $\mathrm{A} / \mathrm{BB}$ ciclo $\mathrm{F}$, mammea $\mathrm{A} / \mathrm{BC}$ ciclo $\mathrm{F}$, isodisparfuram $\mathrm{A}$, mammea $\mathrm{A} / \mathrm{AA}$ ciclo $\mathrm{F}$ e mammea $\mathrm{A} / \mathrm{AB}$ ciclo $\mathrm{F}^{38}$.

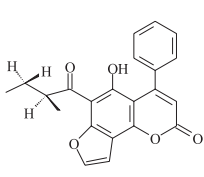

34

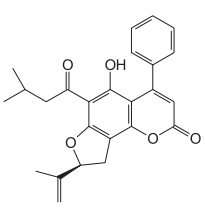

36

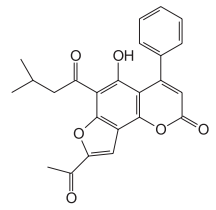

35

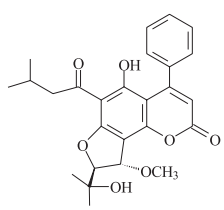

37

\section{Calophyllum thwaitesii}

Das raízes desta planta foram isoladas as substâncias: dimetilcalaxantona, trapezifolixantona, thwaitesixantona, calothwaitesixantona, 6-desoxi-y-mangostin, ácido calozelânico, friedelina, $\beta$-sitosterol e uma nova xantona identificada como 11,12diidro-thwaitesixantona $(\mathbf{3 8})^{39}$.

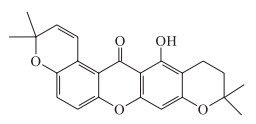

Calophyllum gracilipes

38

Das folhas da C. gracilipes foi isolado um triterpeno inédito identificado como gracilipeno (39), além de outros triterpenos e esteróides conhecidos, incluindo friedelina, lupeol, lupenona, $\beta$-sitosterol, estigmasterol, 3- $\beta$-hidroxi-30-norlupan-20-ona, lupano-3- $\beta$-20-diol, ácido (20R)-3- $\beta$-hidroxilupan-29-óico, ácido betulínico e esqualeno ${ }^{40}$.

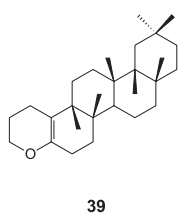

\section{Calophyllum moonii}

C. moonii é uma árvore endêmica da zona úmida do Sri Lanka, cujos principais metabólitos das folhas e caules se constituem de 
xantonas, piranocumarinas, ácidos cromânicos, triterpenóides e esteróides. Das raízes desta espécie foram isolados os compostos dombaquinaxantona (40), calozeilanoxantona (41), friedelina, taraxerol e sitosterol ${ }^{41}$.

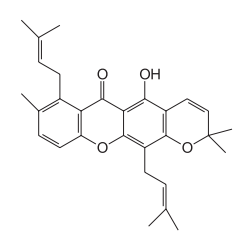

40

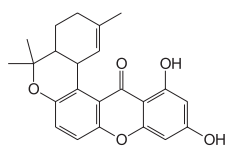

41

\section{Calophyllum cordato-oblongum}

Nesta espécie predominam compostos como xantonas, cumarinas e triterpenóides, tanto nas folhas como os galhos, sendo os principais compostos isolados: cordatolide A, B, oblongulide, ácido cordato-oblôngico (42-45), ácido isocordato-oblôngico, fridelina, canofilol e sitosterol ${ }^{41,42}$.<smiles>[R9]C1C([2H])C(C)C2OC(=O)CC(C)C2C2OC(C)(C)C=CC12</smiles>

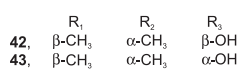

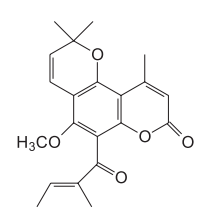

44

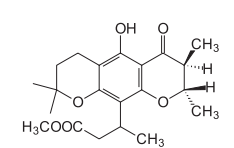

45

\section{Calophyllum panciflorum}

Da $C$. panciflorum algumas substâncias inéditas foram isoladas, incluindo 2 novos biflavonóides, denominados de pancibiflavonol (46) e garcinianina (47), juntamente com GB-2, GB-1, GB-2 A, GB-1A e GD-IV ${ }^{43}$.

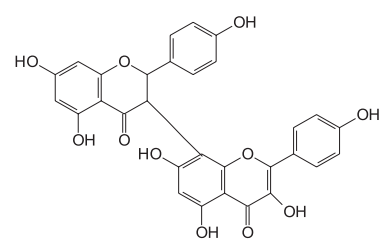

46

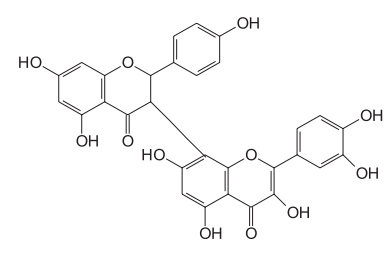

47

\section{Calophyllum caledonicum}

Do extrato de diclorometano da C. caledonicum foram isoladas várias xantonas, sendo as caledonixantona A, B, C, D, E, F (48-53) inéditas. Outras xantonas isoladas foram 7-hidroxi-8metoxixantona; 7,8-dimetoxixantona, 6-hidroxi-5-metoxixantona,7hidroxi-5,6-dimetoxixantona, deidrocicloguanandina. As caledonixantonas $\mathrm{E}$ e $\mathrm{F}(\mathbf{5 2}, \mathbf{5 3})$ foram efetivas contra os fungos A. fumigatus e C. albicans $^{17,44}$.

Em outro estudo com o extrato diclorometano das folhas, foram isoladas duas novas xantonas substituídas, denominadas de caledol e dicaledol, as quais apresentaram atividade antifúngica, especialmente contra A. fumigatus ${ }^{43}$.

As xantonas caloxantona $\mathrm{C}$, dimetilcalabaxantona, calothwaitesixantona, calozeyloxantona e dombakinaxantona, isoladas desta espécie, mostraram atividade antimalárica, sendo ainda mais potentes contra Plasmodium falciparum resistente à cloroquina, quando modificadas quimicamente pela trioxigenação e prenilação das xantonas, demonstrando a relação entre atividade antimalárica e estrutura química destes compostos ${ }^{46}$.

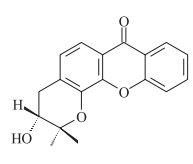

48

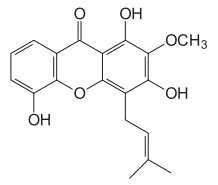

51

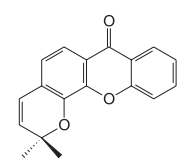

49

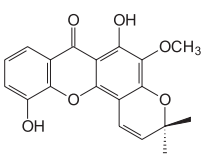

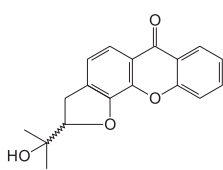

50

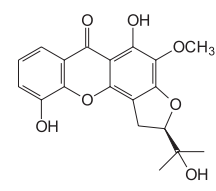

\section{Calophyllum mucigerum}

Estudos com as cascas de C. mucigerum possibilitaram o isolamento de uma nova cumarina, denominada de mucigerina, além da xantona cudraxantona $\mathrm{C}$, friedelina e estigmasterol. O extrato bruto desta espécie foi testado contra a linhagem de células CDM.SS, um tipo de leucemia, onde o extrato apresentou atividade, além de ter toxicidade moderada contra a larva do Aedes aegyti ${ }^{47}$.

\section{Calophyllum teysmannii Miq. Var}

Das raízes desta planta foram isoladas algumas xantonas: 1,2,8trimetoxixantona, 6-hidroxi-1,2,5,7-tetrametoxixantona, ácido teysmanico, 7-hidroxi-1,2,8-trimetoxixantona, 6-hidroxi-1,2,5trimetoxixantona, 2-carbometoxi-6-metoxixantona, 3,8-diidroxi1,2,4-trimetoxixantona, 3-hidroxi-2,4-dimetoxi-xantona, 1,7diidroxi-3-metoxixantona e 2-hidroxixantona ${ }^{48,49}$.

\section{Calophyllum venulosum}

Foram isolados das folhas desta planta dois novos biflavonóides, denominados de piranoamentoflavona 7-metil éter e piranoamentoflavona 4'-metil éter ${ }^{50}$.

\section{Calophyllum polyanthum}

Do extrato etanólico das sementes desta espécie foram isolados duas novas piranocumarinas diastereoisoméricas denominadas de calopolyanolidas $\mathrm{C}$ e $\mathrm{D}$ além dos compostos: calopolyanolida A, B; calanolida $\mathrm{E}_{2}$; 5,7,3',4-tetraidroxiisoflavona; ácido 3,4diidroxibenzóico; ácido 3,4,5-triidróxibenzóico; ácido 2-hidroxi4-metoxibenzóico; $\beta$-sitosterol; $\beta$-daucosterol; ácido 3,5-diidroxi4-metoxibenzóico; ácido 3,5-dimetoxibenzóico; ácido ursólico ${ }^{51}$.

\section{Calophyllum blancoi}

Do extrato acetônico dos frutos desta espécie foram isolados derivados de piranocromanonas denominados de ácido apetalico, 
ácido isoapetalico, ácido metil-éster apetalico, ácido 5-O-acetato apetalico, ácido 5-O-acetato isoapetalico, além do derivado diidrocumarínico denominado de isorecedensolida. As piranocromanonas substituídas apresentaram efeito contra as linhagens $\mathrm{KB}$ e Hela de células tumorais humanas ${ }^{52}$. Das raízes desta planta foram isoladas três novas piranoxantonas, denominadas blancoxantona (54), acetilblancoxantona (55) e 3-hidroxiblancoxantona (56), juntamente com as xantonas piranojaucaeubin e caloxantona ${ }^{53}$.

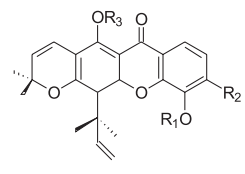

54, $R_{1}=H, R_{2}=H, R_{3}=H$

$55, R_{1}=A c, R_{2}=H, R_{3}=H$

56, $\mathrm{R}_{1}=\mathrm{H}, \mathrm{R}_{2}=\mathrm{OH}, \mathrm{R}_{3}=\mathrm{H}$

\section{Calophyllum enervosum}

A C. enervosum é uma árvore frondosa que chega a medir até $33 \mathrm{~m}$ de altura. Recentemente foi demonstrado que as cascas do caule desta planta, coletadas na Indonésia, possuem efeitos antibacterianos, os quais parecem estar relacionados com a presença de uma nova cetona poliisoprenilada, denominada enervosanona (57). Os mesmos autores também evidenciaram a presença de outras substâncias conhecidas: osajaxantona, cambogina e epicatequina ${ }^{54}$.

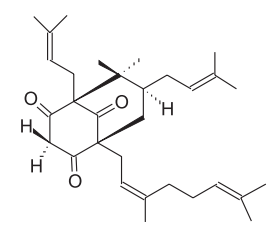

57

\section{CONSIDERAÇÕES FINAIS}

Os estudos químicos e biológicos relatados na literatura do gênero Calophyllum e os resultados obtidos pelo NIQFAR (Núcleo de Investigações Químico-Farmacêuticas) ou em parceria com outras instituições, com a espécie $C$. brasiliense, demonstram a constituição química distinta entre as espécies e também entre as diferentes partes de das plantas, com predominância de metabólitos derivados de xantonas, cumarinas, flavonóides e terpenos, sendo em sua maioria ativos nos vários ensaios biológicos a que foram submetidos.

Alguns compostos chamam a atenção pelas atividades anti-HIV e citotóxicas, além da expressiva atividade antinociceptiva evidenciada por determinados compostos e frações, abrindo perspectivas para novos estudos, a fim de comprovar cientificamente tais efeitos e também os mecanismos de ação envolvidos, podendo gerar protótipos para obtenção de novos fármacos ou fitofármacos.

Estudos que relacionaram estrutura química versus atividade biológica detectaram diferentes atividades e potência entre os compostos analisados, sendo que, geralmente, os compostos com maior grau de substituição e lipossolubilidade apresentaram efeitos biológicos mais relevantes, principalmente citotóxicos e antivirais $^{20,52}$. Estes efeitos demonstram que possíveis modificações estruturais nas moléculas isoladas das espécies de Calophyllum poderão propiciar a descoberta de compostos ainda mais relevantes para a terapêutica.

No entanto, deve-se ressaltar que a maioria dos trabalhos encontrados na literatura não relata estudos toxicológicos in vitro e/ ou in vivo, sendo que tais avaliações são necessárias para continuidade das investigações químico-farmacológicas, especialmente quando se objetiva a formulação de um novo agente medicinal.

Finalmente, pode-se afirmar que o gênero Calophyllum consiste em uma riquíssima fonte de novos compostos químicos, em sua maioria com expressiva atividade biológica, e que a espécie $C$. brasiliense, abundante no Brasil, e com potencial terapêutico comprovado em vários estudos experimentais, pode vir a se tornar um novo fitoterápico genuinamente brasileiro.

Tabela 1. Atividade biológica e principais classes de substâncias de plantas do gênero Calophyllum

\begin{tabular}{|c|c|c|c|}
\hline Espécie & Atividade biológica & Principais classes de substâncias & Ref. \\
\hline C. brasiliense & $\begin{array}{l}\text { analgésica, antiviral, antiulcerogênica, } \\
\text { anticâncer, antibacteriana, moluscicida }\end{array}$ & $\begin{array}{l}\text { terpenos, cumarinas, xantonas, cromanonas, } \\
\text { flavonóides, triterpenos }\end{array}$ & $18-24,26-29$ \\
\hline C. fragrans & - & xantonas & 31 \\
\hline C. inophyllum & $\begin{array}{l}\text { antitumoral, antiviral, } \\
\text { citotóxica, antibacteriana }\end{array}$ & cumarinas, xantonas, triterpenos & $15,33-37$ \\
\hline C. dispar & - & cumarinas & 38 \\
\hline C. thwaitesii & - & xantonas, terpenos & 39 \\
\hline C. gracilipos & - & triterpenos & 40 \\
\hline C. moonii & - & xantonas, cumarinas, triterpenos, esteróides & 41 \\
\hline C. cordato-oblogum & - & xantonas, biflavonóides & 41,42 \\
\hline C. panciflorum & - & xantonas, biflavonóides & 43 \\
\hline C. caledonicum & antifúngica, antimalárica & xantonas & $44-46$ \\
\hline C. mucigerum & antileucêmica, inseticida & xantonas, cumarinas & 47 \\
\hline C. teysmannii & - & xantonas & 48,49 \\
\hline C. venulosum & - & biflavonóides & 50 \\
\hline C. polyanthum & - & cumarinas, ácidos benzóicos & 51 \\
\hline C. blancoi & - & cromanonas, xantonas & 52,53 \\
\hline C. enervosum & antibacteriana & xantonas, cetonas & 54 \\
\hline
\end{tabular}




\section{AGRADECIMENTOS}

À FAPESC/SC, ao CNPq e à ProPPEC/UNIVALI pelo auxílio financeiro.

\section{REFERÊNCIAS}

1. Cechinel Filho, V.; Yunes, R. A. Em Plantas medicinais sob a ótica da química medicinal moderna; Yunes, R. A.; Calixto, J. B., eds.; Argos Ed. Universitária: Chapecó, 2001.

2. Yunes, R. A.; Pedrosa, R. C.; Cechinel Filho, V.; Quim. Nova 2001, 24, 147.

3. Maciel, M. A. M.; Pinto, A. C.; Veiga Jr, V. F.; Quim. Nova 2002, 25, 429.

4. Niero, R.; Malheiros, A.; Bittencourt, C. M. S.; Biavatti, M. W.; Leite, S. N.; Cechinel Filho, V. Em Ciências farmacêuticas. Contribuições ao desenvolvimento de novos fármacos e medicamentos; Bresolin, T. M. B.; Cechinel Filho, V., eds.; Ed. UNIVALI: Itajaí, 2003.

5. Simões, C. M. O.; Schenkel, E. P.; Gosmann, G.; Mello. J. C. P.; Mentz, L. A.; Petrovick, P. R.; Farmacognosia: da planta ao medicamento, $4^{\mathrm{a}} \mathrm{ed}$. UFRGS:Porto Alegre/Florianópolis, 2002.

6. Kinghorn, A. D.; Pharmacother. 2002, 3, 77.

7. Hostettmann, K.; Queiroz, E. F.; Vieira, P. C.; Princípios ativos de plantas superiores, Ed. UFSCar: São Carlos, 2003, cap. 1.

8. Heinrich, M.; Phytother. Res. 2000, 14, 479.

9. Cox, P. A.; Heinrich, M.; Pharmac. News 2001, 8, 55.

10. Capasso, R.; Fitoterapia 2000, 71, 58.

11. Rates, S. M. K.; Toxicon 2000, 39, 603.

12. Oliveira, J. C.; Resumos do $17^{\circ}$ Simpósio de Plantas Medicinais do Brasil, Fortaleza, Brasil,1994.

13. Dharmaratne, H. R.; Perera, D. S. C.; Marasinghe, G. P. K.; Jamie, J.; Phytochemistry 1999, 51, 111.

14. Reyes-Chilpa, R.; Estrada-Muniz, E.; J. Chem. Ecol. 1997, 23, 1901.

15. Ali, M. S.; Mahmud, S.; Perveen, S.; Ahmad, V.; Rizwani, G.; Phytochemistry 1999, 50, 1385.

16. Mckee, T. C.; Covington, C. D.; J. Nat. Prod. 1998, 61, 1252.

17. Morel, C.; Seraphin, J.; Oger, J. M.; Litaudon, M.; Sevenet, T.; Richomme, P.; Bruneton, J.; J. Nat. Prod. 2000, 63, 1471.

18. Silva, K. L.; Santos, A. R.; Mattos, P. E.; Yunes, R. A.; Delle-Monache, F.; Cechinel Filho, V.; Therapie 2001, 56, 431.

19. Sartori, N. T.; Canepelle, D.; Sousa Jr., P. T.; Martins, D. T. O.; J. Ethnopharmacol. 1999, 67, 149.

20. Reyes-Chilpa, R.; Estrada-Muniz, E; Apan, T. T.; Amekraz, B.; Aumelas, A.; Jankowski, C. K.; Vazquez-Torres, M.; Life Sci. 2004, 75, 1635.

21. Ito, C.; Itoigawa, M.; Mishina, Y.; Cechinel Filho, V.; Mukainaka, T.; Tokuda, H.; Nishino, H.; J. Nat. Prod. 2002, 65, 267.

22. Ito, C.; Itoigawa, M.; Mishina, Y.; Cechinel Filho, V.; Enjo, F.; Tokuda, H.; Nishino, H.; Furukawa, H.; J. Nat. Prod. 2003, 66, 368.

23. Cottiglia, F.; Dhanapal, B.; Sticher, O.; Heilmann, J.; J. Nat. Prod. 2004, 67, 537.

24. Huerta-Reyes, M.; Basualdo, M. C.; Abe, F.; Jimenez-Estrada, M.; Soler, C.; Reyes-Chipa, R.; Biol. Pharm. Bull. 2004, 27, 1471.

25. Pretto, J. B.; Cechinel Filho, V.; Noldin, V. F.; Sartori, M. R. K.; Isaias, D. E. B.; Bella Cruz, A.; Z. Naturforsch. 2004, 56, 657.

26. Stout, G.; Krahn, M. M.; Breck, G. D.; Tetrahedron Lett. 1968, 29, 3285.
27. Isaias, D. E. B.; Niero, R.; Noldin, V. F.; Campos-Buzzi, F. De; Yunes, R. A.; Delle-Monache, F.; Cechinel Filho, V.; Die Pharmazie 2004, 59, 879.

28. Kimura, S.; Ito, C.; Jyoko, N.; Segawa, H.; Kuroda, J.; Okada, M.; Adachi, S.; Nakahata, T.; Yuasa, T.; Cechinel Filho, V.; Furukawa, H.; Maekawa, T.; Int. J. Cancer 2005, 113, 158.

29. Ravelonjato, B.; Kunesch, N.; Poisson, J. E.; Phytochemistry 1987, 26, 2973.

30. Gasparotto Jr., A.; Brenzan, M. A.; Piloto, I. C.; Cortez, D. A. G.; Nakamura, C. V.; Dias Filho, B. P.; Rodrigues Filho, E.; Ferreira, A. G.; Quim. Nova 2005, 28, 575.

31. Locksley, H. D.; Murray, I. G.; Phytochemistry 1969, 8, 927.

32. Potti, G. R.; Kurup, P. A.; Indian J. Exp. Biol. 1970, 8, 39.

33. Linuma, M.; Tosa, H.; Tanaka, T.; Yonemorit, S.; Phytochemistry 1994, 35 , 527

34. Jantan, I. B.; Jalil, J.; Warif, N. M. A.; Pharm. Biol. 2001, 39, 243.

35. Itoigawa, M.; Ito, C.; Tan, H. T. W.; Kuchide, M.; Tokuda, H.; Nishino, H.; Furukawa, H.; Cancer. Lett. 2001, 169, 15.

36. Yimdjo, M. C.; Azebaze, A. G.; Nkengfack, A. E.; Meyer, A. M.; Bodo, B.; Fomum, Z. T.; Phytochemistry 2004, 65, 2789.

37. Laure, F.; Herbette, G.; Faure, R.; Bianchini, J. P.; Raharivelomanana, P.; Fogliani, B.; Magn. Reson. Chem. 2004, 43, 65.

38. Guilet, D.; Helesbeux, J. J.; Seraphin, D.; Richomme, P.; Bruneton, J.; J. Nat. Prod. 2001, 64, 563.

39. Dharmaratne, H. R. W.; Wanigasekera, W. M.; Phytochemistry 1996, 42, 249

40. Cao, S. G.; Sim, K.Y.; Goh, S. H.; Xue, F.; Mak, T. C. W.; Tetrahedron Lett. 1997, 38, 4783.

41. Dharmaratne, H. R. W.; Wijesinghe, W. M. N. M.; Phytochemistry 1997, 46, 1293.

42. Dharmaratne, H. R.; Sajeevani, M.; Marasinghe, G. P. K.; Ekanayake, E. M. H.G. S.; Phytochemistry 1998, 49, 995.

43. Ito, C.; Itoigawa, M.; Miyamoto, Y.; Rao, K.S.; Takayasu, J.; Mukainaka, T.; Tokuda, H.; Nishino, H.; Furukawa, H.; J. Nat. Prod. 1999, 62, 1668.

44. Morel, C.; Seraphin, D.; Teyrouz, A.; Larcher, G.; Bouchara, J. P.; Litaudon, M.; Richomme, P.; Bruneton, J.; Planta Med. 2002, 68, 41.

45. Oger, J. M.; Morel, C.; Helesbeux, J. J.; Litaudon, M.; Seraphin, D.; Dartiguelongue, C.; Larcher, G.; Richomme, P.; Duval, O.; Nat. Prod. Res. 2003, 17, 195.

46. Hay, A. E.; Helesbeux, J. J.; Duval, O.; Labaied, M.; Grellier, P.; Richomme, P.; Life Sci. 2004, 75, 3077.

47. Ee, G. C.; Ng, K. N.; Taufiq-Yap, Y. H.; Rahmani, M.; Ali, A. M.; Muse, R.; Nat. Prod. Res. 2004, 18, 123.

48. Kijjoa, A.; Gonzalez, M. J.; Pinto, M. M.; Anantachoke, C.; Herz, W.; Phytochemistry 2000, 55, 833.

49. Kijjoa, A.; Gonzalez, M. J.; Afonso, C. M.; Pinto, M. M.; Anantachoke, C.; Herz, W.; Phytochemistry 2000, 53, 1021.

50. Cao, S. G.; Sim. K. Y.; Goh, S. H.; Nat. Prod. Lett. 2001, 15, 291.

51. Ma, C. G.; Chen, B.; Qi, H. Y.; Li, B. G.; Zhang, G. L.; J. Nat. Prod. 2004, 67, 1598.

52. Shen, Y. C.; Wang, L. T.; Khalil, A. T.; Kuo, Y. H.; Chem. Pharm. Bull. 2004, 52, 402 .

53. Shen, Y. C.; Wang, L. T.; Khalil, A. T.; Chiang, L. C.; Cheng, P. W.; Chem. Pharm. Bull. 2005, 53, 244.

54. Taher, M.; Idris, M. S.; Ahmad, F.; Arbain, D.; Phytochemistry 2005, 66 , 723 